\title{
Estereotipos de género en educación física
}

\section{Gender stereotypes in physical education}

\author{
Juan Carlos Pastor-Vicedoㄹ, Adrián Sánchez-Oliva², Jonatan Sánchez-Blanchart² y Jesús Martínez-Martínez ${ }^{3 *}$ \\ ${ }^{1}$ Profesor Facultad de Educación de Albacete. Universidad de Castilla-La Mancha. Albacete (España). \\ ${ }^{2}$ Miembro grupo de trabajo en Educación Física de la Facultad de Educación de Toledo. Universidad de Castilla-La Mancha. Toledo (España). \\ ${ }^{3}$ Profesor Facultad de Educación de Toledo. Universidad de Castilla-La Mancha. Toledo (España).
}

\begin{abstract}
Resumen: La escuela y en particular el área de Educación Física, puede ser uno de los escenarios donde, bajo el denominado currículum oculto, se pongan de manifiesto actitudes estereotipadas y cargadas de prejuicios desde el punto de vista deportivo y motor. El objetivo principal de nuestro estudio fue identificar la percepción de los escolares de Educación Primaria

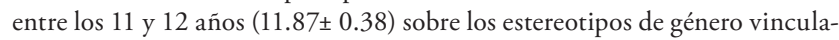
dos con la actividad física y el deporte, a partir de subestereotipos asociados al tipo de práctica, respecto al profesorado y a clima de clase. Los principales resultados muestran la presencia de estereotipos relacionados con el rendimiento, el autoconcepto y el trato por parte del profesorado. Desde la escuela y desde el área de Educación Física se debe tener muy presente estas situaciones que debemos reorientar para lograr una verdadera escuela inclusiva y sociedad que permita la igualdad de oportunidades.

Palabras clave: Estereotipos, género, Educación Primaria, Educación Física.
\end{abstract}

Abstract: The school, and the Physical Education may become as one of the setting where with the so-called hidden curriculum, attitudes loaded with prejudices, in terms of gender stereotyped, could be shown. The main objective of our study was to identify the perception of primary school students between 11 and 12 years old (11.87 \pm 0.38$)$ about gender stereotypes linked to physical activity and sport, based on sub-stereotypes associated with the type of practice, regarding the teaching staff and classroom climate. The main results show the presence of stereotypes related to performance, self-concept and treatment by teachers. From the school and from the area of Physical Education, we must bear in mind these situations that we must reorient to achieve a true inclusive school and society that allows equal opportunities.

Keywords: Stereotypes, gender, Primary Education, Physical Education.

\section{Introducción}

Vivimos en una sociedad caracterizada ante todo por la diversidad cultural, en la que numerosos conflictos personales y sociales desestabilizan constantemente la posibilidad de convivir de manera armónica. La necesidad de encontrar soluciones que permitan el entendimiento entre géneros, entre otros asuntos, es fundamental para paliar las situaciones enfrentadas que surgen en ésta.

Inicialmente, el concepto de género hace mención al conjunto de características y roles que la sociedad asigna a los seres humanos y que éstos asumen como propio. El género, es fundamentalmente una construcción social, es decir, no es una característica natural del ser humano, y esta varía entre grupos sociales y épocas. Este término es el resultado de la construcción a través de relaciones entre hombres y mujeres, que desde la infancia se van agrupando para formar el concepto al cual se siente identificado. El género es aquello que la sociedad asigna a lo 'femenino' y a lo 'masculino' de las cosas, es decir, se considera como algo dinámico; el sexo, por el contrario, es estático (Lamas, 2000). El término "sexo" hace referencia a las diferencias físicas entre el hombre y la mujer, las cuales, aparecen desde el nacimiento de los seres humanos,

Dirección para correspondencia [Correspondence address]: Jesús Martinez-Martínez. Universidad de Castilla-La Mancha. Facultad de Educación de Toledo. Avda. Carlos III, s/n Universitario s/n. 45004. Toledo (España).E-mail: Jesus.mmartinez@uclm.es y van avanzando aún más en su posterior crecimiento hacia la plena madurez. Este concepto determina las características genéticas, hormonales, fisiológicas y funcionales que diferencian a cada ser humano.

Estas diferencias en la construcción social, simbolizan todo aquello que es distinto a lo que conocemos, pero que no por ello debe ser considerado inferior o negativo, sino que se han convertido en prejuicios estableciendo un estereotipo al respecto, lo que siguiendo a autores como Frömel, Formánková y Sallis (2002) ha condicionado aspectos como el acceso a determinadas funciones sociales reservadas en la mayoría de las ocasiones al ámbito masculino respecto al femenino en detrimento de éstas últimas, por ejemplo: política, religión, responsabilidades empresariales..., espacios que podrían pensarse como de poder y de toma de decisiones.

Blández, Fernández y Sierra (2007) señalan que, del estudio de los estereotipos de género vinculados con la actividad física, la línea definitoria más inmediata ha sido la identificación de dos grandes conjuntos o rasgos, los instrumentales, asociados con la masculinidad; y los afectivos-expresivos, asociado a rasgos femeninos, con su consecuente derivación en las tipologías estereotipadas de "hombre físicamente activo" y "mujer físicamente pasiva".

Los seres humanos viven un proceso de socialización muy distinto durante su infancia, adolescencia y madurez, donde se adquieren formas de pensar, de relacionarse, de actuar, de 
divertirse, de trabajar, etc. Toda esta diversidad son formas generales de convivir con la sociedad en el mundo, de vivir determinados por diversos factores, que van dando forma a la personalidad. Dentro de dicha personalidad creamos poco a poco unos estereotipos que condicionan todas las acciones que podemos realizar como seres humanos. Así pues, donde se ha manifestado de manera muy clara es en el ámbito físico desde el punto de vista deportivo y motor (la fuerza, la velocidad, la competitividad, etc.) dónde han servido a lo largo de la historia como la excusa perfecta para elaborar las diversas teorías sobre la supuesta inferioridad de quienes no son portadores de esos rasgos y, como consecuencia, para practicar todo tipo de actitudes discriminatorias.

La escuela no es ajena a esta realidad y así autores como Williams y Best (1990), ya señalaban que los estereotipos en el campo educativo podemos definirlos como creencias de género compartidos socialmente, que traen consigo un impacto entre las relaciones de chicas y chicos. En el ámbito de la educación pueden provenir de diferentes fuentes como pueden ser: estereotipos del entorno y afectan al pensamiento del alumno (anuncios publicitarios, sucesos de discriminación de la sociedad, estereotipos familiares, etc.); estereotipos del profesorado hacia los alumnos, que provocan actitudes discriminatorias entre el alumnado; estereotipos de los propios alumnos que provienen de su propio pensamiento, y son el resultado de la suma y combinación de los estereotipos anteriormente mencionados.

En esta misma línea, la influencia de los estereotipos se ha estudiado desde distintas perspectivas, tal y como exponen Blández et al. (2007) y Alvariñas y Novoa (2015), considerándose entre otras, las relacionadas con el interés y la valoración de la clase y del profesorado, el análisis de género de cómo el profesorado desarrolla su enseñanza, las representaciones del profesorado y sus atribuciones de género, o las creencias de los escolares en relación con tipificaciones de género. En este contexto, la Educación Física (EF) dentro del entorno escolar parece la más apropiada para la visualización de dichos comportamientos desde el punto de vista motor. A lo largo del tiempo se han ido transmitiendo generación tras generación una serie ideas convertidas en prejuicios, que han hecho que, en la actualidad, sigan reflejándose diferencias entre chicos y chicas dificultando un desarrollo tolerante, respetuoso, cooperativo, y en definitiva, coeducativo e inclusivo.

En numerosas ocasiones, tal y como mostraban Valdivia, López, Lara y Zagalaz (2012) esto conlleva que las chicas sean consideradas un problema desde el punto de vista del rendimiento deportivo y por ello es fundamental que el profesorado de EF conozca realmente que es la coeducación para poder trabajar con una metodología adecuada. Para estos autores, en el campo de la EF parece haber controversia a la hora de definir los términos, debiendo distinguir entre coeducación e igualdad de género, afirmando que la coeducación, debe promover la igualdad de trato entre sexos, no aceptando el modelo universal masculino y desarrollando las cualidades de cada individuo, por lo que la igualdad es necesaria para poder desarrollar la coeducación, teniendo en cuenta que se trabaja con dos sexos diferentes que conviven en una realidad social común.

Otra de las cuestiones asociadas al campo educativo reside en el análisis de cuál es la metodología de trabajo utilizada por parte del profesorado, ya que autores como Soler (2006) y Vázquez, Fernández-García y Ferro (2000) indicaban que el comportamiento del alumnado y las creencias del profesorado siguen siendo muy estereotipadas, pudiéndose generar múltiples posibilidades de desigualdad. Siguiendo a estos autores, estas situaciones se dan en cuestiones como la distribución del espacio y el material, las interacciones entre chicos y chicas, y el tipo de estrategias didácticas que utilizan los docentes, siendo necesario tal y como señalan GómezMármol y De la Cruz Sánchez (2013) que los profesores se convenzan de la importancia de este fenómeno de modo que incluyan mecanismos en sus clases para prevenir o evitar esta situación. Los prejuicios asociados a estas situaciones hacia las niñas también han privado a los niños a participar en diferentes actividades que como identifica Valdivia et al. (2012) son "propias de niñas". En España, son varios los estudios que han demostrado la permanencia de prejuicios sexistas entre el profesorado y el efecto que estos puede tener en la formación del alumnado, Piedra, García-Pérez, Rebollo y Ries (2011); Valdivia et al. (2012); Castillo, Romero, González y Campos (2012).

El alumnado parece tener admitido su rol y lo reproduce sin cuestionarlo, lo que evidencia la necesidad de atención y estudio. El presente trabajo aborda el estudio de los estereotipos y desigualdades de género relacionados con la actividad física y el deporte que existen dentro del entorno escolar, desde la perspectiva de la escuela como agente de transmisión cultural, y de cómo ésta puede ser uno de los soportes principales de la igualdad de oportunidades de ambos géneros. De esta manera se pretende promover una mayor equidad y acceso a los beneficios que la actividad física proporciona en la edad escolar, tanto sobre el desarrollo personal como sobre la calidad de vida de los adolescentes y la mejora de los factores asociados a su bienestar, pues existen numerosas evidencias de las diferencias en las oportunidades y dedicación hacia la actividad física, con los consiguientes problemas de adherencia hacia la misma y como consecuencia hacia salud, concretamente por el sector femenino (WHO, 2018; Fernández, Canet y Giné-Garriga, 2017; López, Lara, Espejo y Cachón, 2016; ALADINO, 2015, Secchi, García, España-Romero y Castro-Piñero, 2014). Está claro el importante papel que en este proceso juega la percepción y las interpretaciones que realiza cada persona, por lo que un paso clave y necesario en el estudio de los estereotipos, es la comprensión de las in- 
terpretaciones que realizan los escolares sobre la cultura de género en la actividad físico-deportiva, tal y como afirman Blández et al. (2007).

En base a este planteamiento inicial, el objetivo principal de nuestro estudio será identificar la percepción que tienen los escolares de Educación Primaria sobre los estereotipos de género vinculados con la actividad física y el deporte, y como objetivos específicos:

- Identificar las concepciones de género de los profesores de educación física.

- Mostrar las diferencias de la percepción del alumnado en la participación de sus compañeros/as.

- Establecer las relaciones que existen entre la representación social de los estereotipos, su percepción subjetiva por las chicas y los chicos, y las manifestaciones concretas que sobre ellos se producen en la escuela.

- Conocer los tipos de estereotipos que pueden tener los alumnos/as analizando diferentes variables que van desde el trato que reciben del profesorado, las notas que se las asignan según sea su sexo, las actitudes del alumnado en la formación de grupos mixtos, el comportamiento en la realización de deportes competitivos en equipos mixtos.

- Reconocer las sensaciones en la ejecución de los contenidos propios de la expresión corporal.

\section{Método}

\section{Muestra}

La muestra está compuesta por $\mathrm{N}=54$ alumnos (27 niños y 27

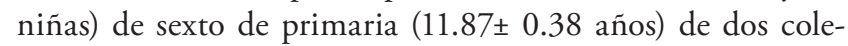
gios públicos de la ciudad de Toledo (España) ubicados en un barrio de la ciudad de nivel socioeconómico medio-bajo. Las características que presenta este grupo son que existe un porcentaje de inmigrantes que, generalmente, presentan retraso educativo por desconocimiento del idioma. Hay alumnado que muestra poco interés, coincidiendo, en la mayoría de los casos, con familias poco implicadas en el proceso educativo de sus hijos. La gran mayoría trabajan bien, aunque el grupo es bastante heterogéneo en cuanto a características psicológicas y físicas, intereses y motivaciones.

\section{Material}

En la investigación de los estereotipos de género en EF que tienen los alumnos es necesario comprobar el pensamiento de estos y como les puede afectar el trato de los docentes dentro de la escuela. El instrumento para visualizar este pensamiento utilizado ha sido el cuestionario, en el cual, se les da opción a responder de forma positiva, negativa, e incluso neutral a la pregunta o situación correspondiente. Este tipo de instru- mento posibilita la reflexión y construcción crítica de una respuesta, ya que les concede más tiempo a la hora de responder, y lo que es más importante es que se trata de una manera de recopilar información de forma anónima, debido a que el alumnado solo se identificará con su género, niño o niña según proceda (Fernández et al., 2007). Así mismo, autores como Alvarińas y Novoa (2015) estudiaron diversas cuestiones relativas al estilo de vida y a pensamientos en relación con la actividad física y el deporte a partir del cuestionario de estilo de vida deportiva con perspectiva de género elaborado por Alvarińas, Fernández-Villarino y López Villar (2009) del cual se extraen ideas comunes a nuestro planteamiento.

El cuestionario elaborado ha sido una adaptación del cuestionario de Percepción de Igualdad-Discriminación en EF (CPIDEF) diseñado por Cervelló, Jiménez, Del Villar, Ramos y Santos-Rosa (2004) que centraba la atención en la percepción del alumnado hacia sus docentes y la igualdad de trato en las sesiones de EF. Dicho test consta de 19 ítems, 10 de los cuales pertenecen a conductas de "igualdad de trato" y se corresponden a parámetros que respetan la igualdad de trato en función del género (p.ej.: "en las clases de EF mi profesor emplea las mismas normas para las alumnas que para los alumnos", "delega responsabilidades tanto en chicas como en chicos"), mientras que los otros nueve ítems corresponden a “discriminación " correspondiente a parámetros discriminatorios (ej. "mi profesor no dedica el mismo tiempo de atención a los chicos que a las chicas", "motiva y anima de forma diferente a los chicos que a las chicas") y aspectos tales como la agrupación, tipo de evaluación, tiempo de atención, participación, expectativas previas de rendimiento y distribución de responsabilidades en chicos y chicas en las clases de EF. También se utilizaron cuestiones relacionadas con la aceptabilidad social de la práctica de actividades físicas y deportivas utilizadas por Fernández et al. (2007) donde se recogía un listado de actividades físico-deportivas donde se solicita que se señale si cada actividad marcada se considera apropiada principalmente para los chicos, o principalmente para las chicas, o es apropiada tanto para los chicos como las chicas.

\section{Procedimiento}

El diseño es de tipo descriptivo - interpretativo. En primer lugar, se realizó una revisión de la literatura al efecto en los buscadores MedLine, SPORTDiscus, y ProQuest utilizando como palabras clave: niños, coeducación, escuela inclusiva, género, educación primaria, tanto en castellano como en lengua inglesa.

A través del cuestionario elaborado al efecto se pretende revisar seis ámbitos: trato del profesorado, evaluación del profesorado, estereotipos en grupos mixtos, estereotipos de deportes de equipo (vinculación masculina), estereotipos sobre expresión corporal (vinculación femenina), y la influencia 
de los medios tomando como referencia la respuesta a las siguientes cuestiones:

- ¿Cuáles son los mecanismos de producción y reproducción de los estereotipos de género en las clases mixtas de educación física?

- ¿Cuáles son las concepciones de género de los profesores de educación física?

- ¿Qué comportamientos y actitudes presentan los profesores en las clases mixtas de Educación Física?

- ¿Qué concepciones de género subyacen en el lenguaje utilizado por alumnos y docentes en las clases mixtas de educación física?

- ¿Qué percepción de trato sobre género tienen los grupos de estudiantes de las clases de educación física mixtas?

- ¿Qué percepción tienen los grupos que cursan el espacio curricular educación física en grupos mixtos sobre sus clases?

Estos cuestionarios, en formato papel y presentados en el aula a través de proyección digital fueron entregados al tutor del curso de $6^{\circ}$ de primaria para su revisión. Cada alumno debía rellenar el cuestionario en el tiempo de duración de la sesión de 45 minutos, para así cualquier duda poderla resolver en el instante. El tipo de respuesta era nominal en una escala de si, a veces y no, donde el alumno solo podía elegir una respuesta válida, respondiendo a cada uno de los cuatro indicadores que describían el propio ítem. Tras rellenar dicho test, se recogió y organizaron los test según el sexo del alumno para facilitar la recopilación de respuestas, para posteriormente analizar los resultados de las respuestas a través de análisis estadístico.

\section{Análisis estadístico}

El análisis de los datos aplicó estadística cuantitativa de carácter descriptivo y análisis de frecuencias. Se realizó un análisis exploratorio de los datos y de los estadísticos descriptivos básicos, calculándose medias, desviaciones típicas, valores máximo y mínimo de las variables y porcentajes. En estos últimos, se han obtenido las distribuciones de frecuencias de las variables consideradas en el estudio. El análisis estadístico se llevó a cabo a través del paquete estadístico SPSS v.24.0.

\section{Resultados}

Los estereotipos analizados a continuación son los que engloban el resto de los estereotipos que forman dicho cuestionario. Además, se analizan los subestereotipos en conjunto en cada estereotipo general.

En la figura 1 se puede observar como los alumnos manifiestan (78\% chicos; $60 \%$ chicas) que el profesorado no muestra diferencias en el trato respecto a ambos géneros, aunque de forma ocasional un 33\% la opinión de las chicas con respecto al $18 \%$ de los chicos opinan que sí. En general, la muestra total $(69 \%)$ nos indica que no se percibe un trato diferente entre géneros por parte del profesorado. En la figura 2 , se puede ver como en los diversos subíndices que componen el estereotipo 1, tampoco se observan diferencias entre géneros ni en el total de la muestra, indicándose valores del $84 \%$ en los chicos y $80 \%$ en las chicas, siendo el $82 \%$ en el total de la muestra.

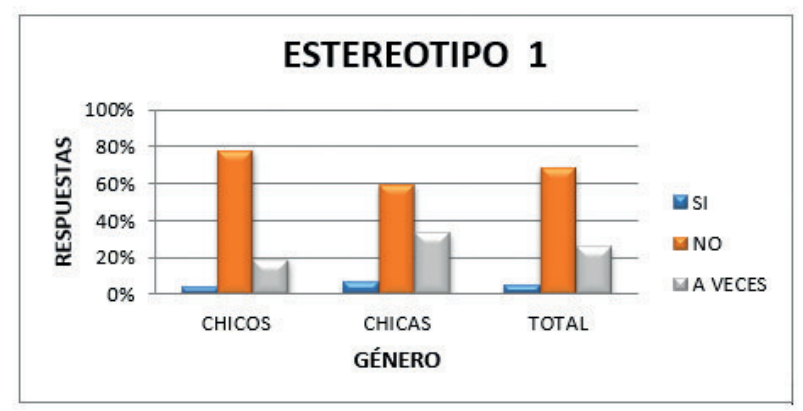

Figura 1. Estereotipo 1. En la escuela, el profesorado de Educación Física trata a los chicos y a las chicas de forma diferente.

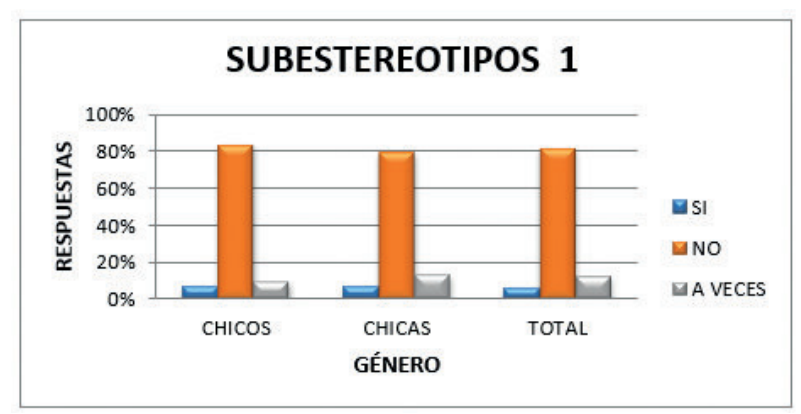

Figura 2. Sub - ítems; distintas actividades, atención, participación, lenguaje y gestos. $(1.1,1.2,1.3,1.4)$.

Los resultados muestran en la figura 3, que el $70 \%$ de los chicos y el $77 \%$ de las chicas niegan este estereotipo, aunque si bien parece apreciarse un incremento en las chicas con respecto a los chicos en la apreciación que hacen al respecto. Igual ocurre cuando observamos los subestereotipos que se corresponden con este estereotipo (figura 4). Los chicos parecen apreciar más igualdad entre la existencia o no de estereotipos de este tipo, debido a que piensan que el profesor ve superiores a los alumnos a nivel de rendimiento, y además, los chicos destacan en las notas. Por el contrario, los alumnos no piensan que se exija diferente esfuerzo entre ambos géneros, ni que se asignen diferentes puestos de responsabilidad, ya que como hemos podido observar en el estereotipo 1, el trato es equitativo.

Por el lado de las chicas, vemos una gran mayoría, con un 
$75 \%$, que indica la presencia de estos estereotipos específicos en su totalidad sin ninguna contrariedad entre ellos. Se puede observar la presencia de este estereotipo en la evaluación de aspectos específicos por parte de su profesorado en el área de EF (58\%), sobre el alumnado que piensan que no, con un $23 \%$.

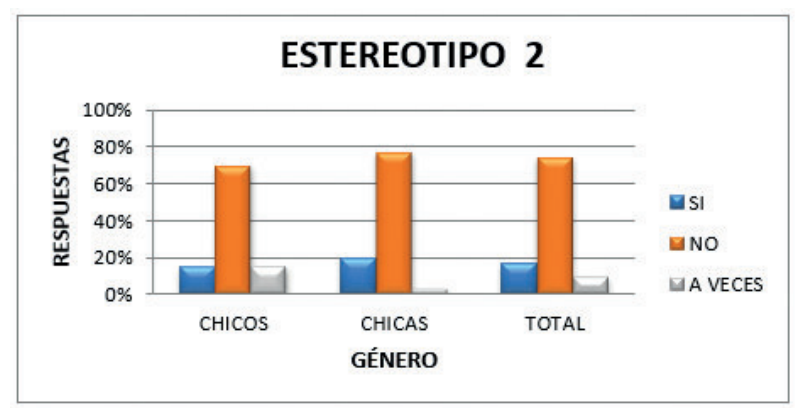

Figura 3. Estereotipo 2. En Educación Física, a veces los profesores y profesoras nos evalúan de forma diferente según seamos chicos o chicas.

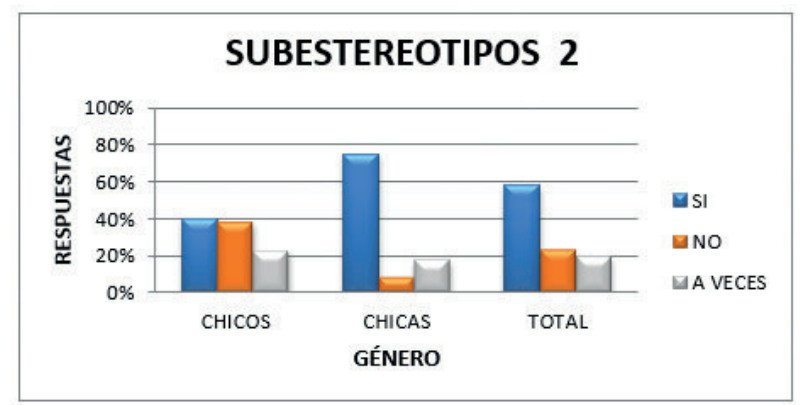

Figura 4. Sub - ítems; diferente rendimiento, esfuerzo, responsabilidad y calificaciones. $(2.1,2.2,2.3,2.4)$.

En la figura 5 y 6 se muestra la existencia de diferencias entre géneros en los grupos mixto, donde los valores parecen mantener una similitud, aunque cuando se observan las valoraciones realizadas en los subapartados que componen el estereotipo general 3, parece existir diferencias entre géneros, siendo las chicas las que sí aprecian ciertas diferencias en los grupos mixtos.

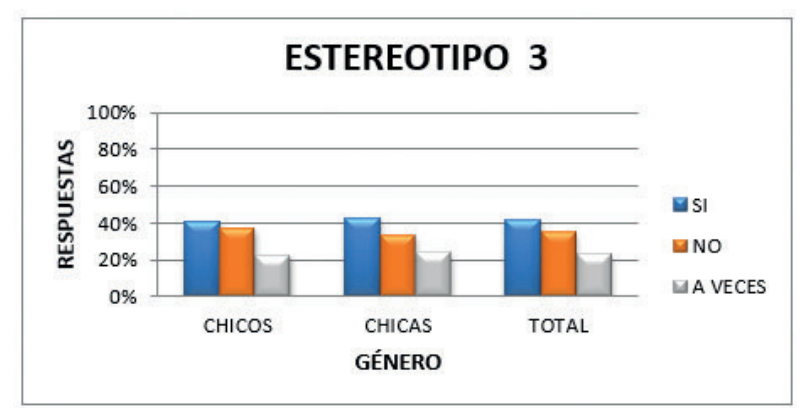

Figura 5. Estereotipo 3. En los grupos mixtos de Educación Física se ven más diferencias entre chicos y chicas.

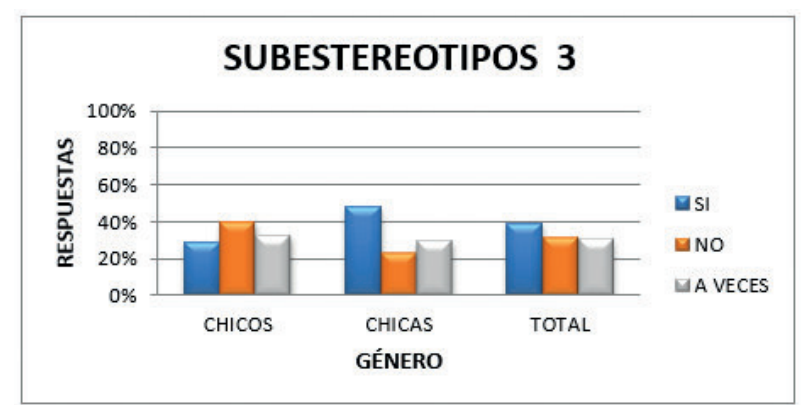

Figura 6. Sub - ítems; distinta organización, rol, rendimiento y complejo. $(3.1,3.2,3.3,3.4)$.

En relación a la práctica y competición de deportes de equipo parece haber diferencias de comportamiento entre ambos sexos, donde un 78\% de los chicos y un 63\% de las chicas opinan así. En general, ambos géneros coinciden, lo que nos indica la claridad con la que los nińos observan las diferencias entre chicos y chicas en los deportes de equipo. En cambio, si analizamos los subestereotipos que lo conforman (figura 8), podemos observar cómo responden de manera diferente. Por un lado, los chicos dicen que no existe discriminación por la superioridad en los deportes de equipos, que existe una total igualdad en la participación según la competitividad y en la falta de capacidad competitiva por parte de las chicas; y por último, una gran mayoría que opina que los géneros no deben ir separados en la actividad física. Por otro lado, las chicas apoyan la idea de la discriminación de los chicos hacia ellas por superioridad en la práctica deportiva; pero niegan su escasa participación, la incapacidad de competición equitativa y la separación de los géneros en las actividades deportivas.

En general, en estos estereotipos específicos tenemos una mayoría que coincide en que no existen diferencias en la práctica de deportes de equipo, con un 57\% frente a un $20 \%$.

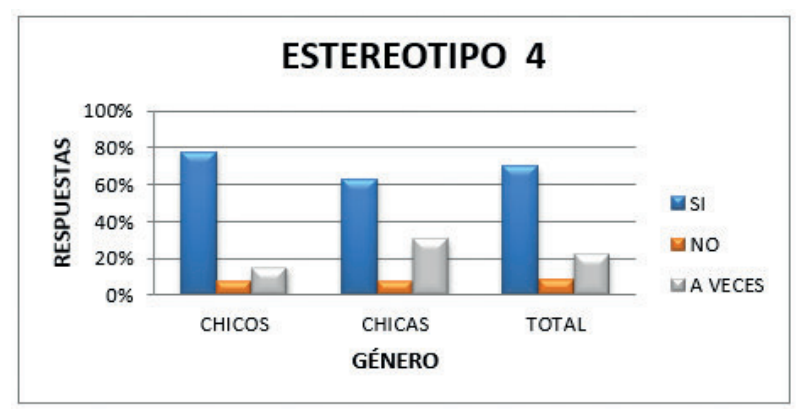

Figura 7. Estereotipo 4. En relación a la práctica y competición de deportes de equipo hay diferencias de comportamiento entre ambos sexos. 


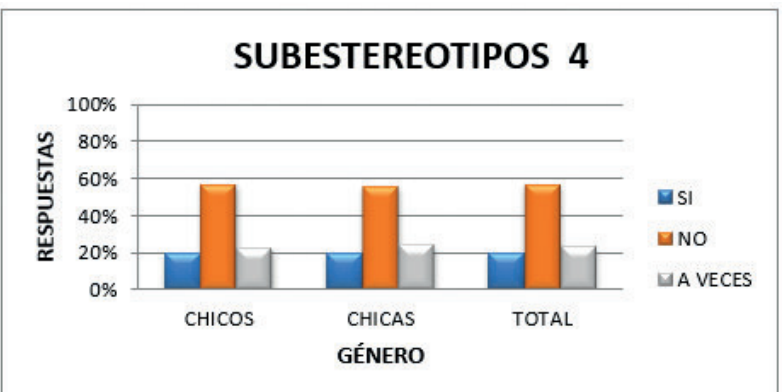

Figura 8. Sub - ítems; distinta superioridad, participación, capacidad de competición y actividades. (4.1, 4.2, 4.3, 4.4).

El análisis del estereotipo relacionado con la expresión corporal y la diferencia de comportamiento entre chicos y chicas (figura 9), nos muestra diferentes porcentajes de respuesta en la percepción que sobre el mismo tienen, ya que tanto los chicos, con un $45 \%$ frente a un $18 \%$, como las chicas, con un $44 \%$ frente a un $27 \%$, están de acuerdo en la presencia de dichas diferencias.

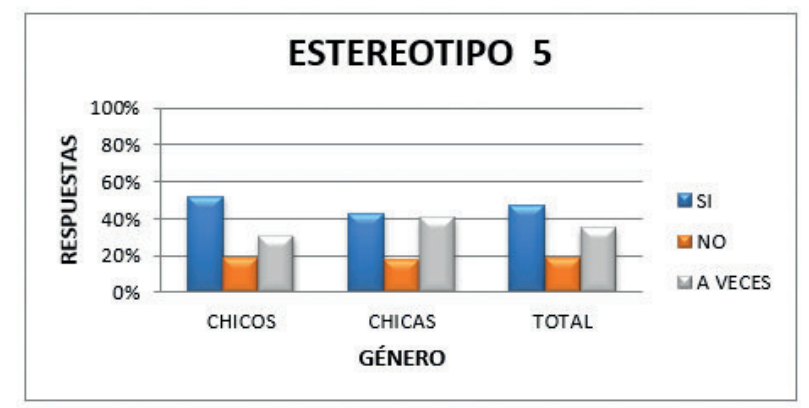

Figura 9. Estereotipo 5. En la ejecución de expresión corporal existen diferencias de comportamiento en los chicos y en las chicas.

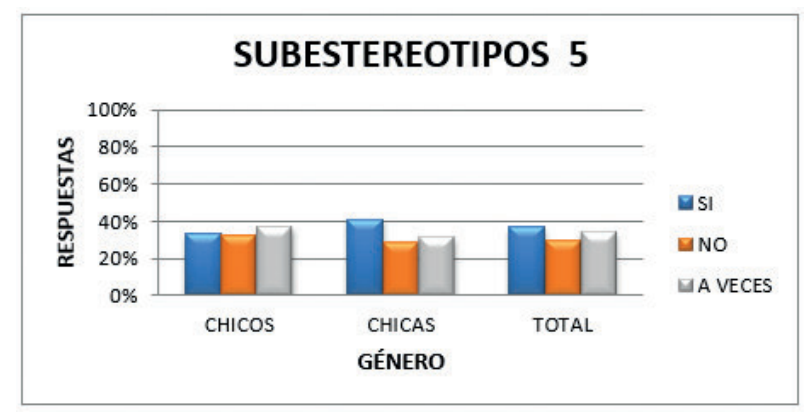

Figura 10. Sub - ítems; diferente protagonismo, expresión corporal, ritmo, organización. $(5.1,5.2,5.3,5.4)$

En los estereotipos específicos relacionados con la expresión corporal (figura 10) obtenemos pequeñas variaciones de la presencia de los mismos, con un $37 \%$ frente a un $29 \%$ que opina lo contrario, y un $34 \%$ que habla de una existencia ocasional de dicho estereotipo. Por un lado, los chicos tienen una opinión muy diversa que provoca que no destaque plenamente ninguna opción, aunque se aprecia la existencia de diferencias de género en la expresión corporal con un 33\%. Por otro lado, las chicas destacan más la presencia de este estereotipo con un $41 \%$ frente a un $28 \%$ que lo niega. Comparando ambos sexos observamos coincidencia en la negación del protagonismo masculino, en el mejor rendimiento de las chicas en el contenido de expresión corporal, y en la preferencia por ejercer la práctica con el propio género. También, podemos observar la opinión clara que tienen las chicas sobre la existencia de dificultad en la expresión corporal y el ritmo que presentan los varones, frente a la igualdad totalitaria de opinión de los chicos sobre su propia dificultad en este contenido.

El análisis de las diferencias de género en los medios de comunicación (figura 11), nos muestra un acuerdo entre ambos géneros. Por un lado, los chicos presencian diferencias en los medios, $44 \%$ frente a un $41 \%$ que piensa que no existen dichas diferencias. Por otro lado, las chicas tienen clarísimo que existen estas diferencias entre géneros en los medios, ya que un $70 \%$ está totalmente de acuerdo en la presencia de tales diferencias, frente a un $8 \%$ que opina lo contrario.

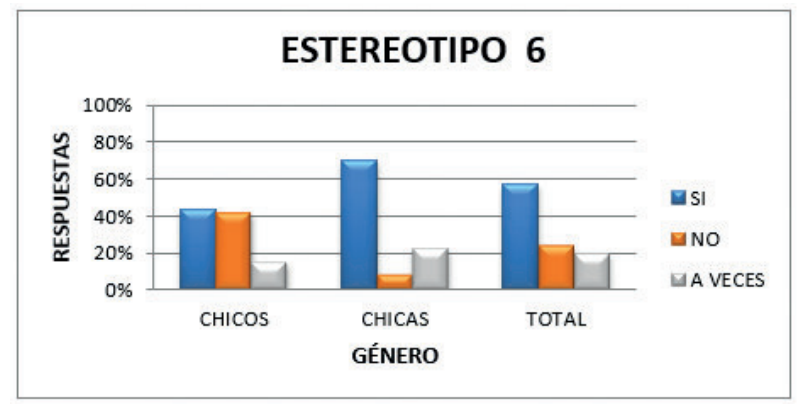

Figura 11. Estereotipo 6.El deporte que aparece en los medios (televisión, revistas, internet, etc.) muestra diferencias entre el hombre y la mujer.

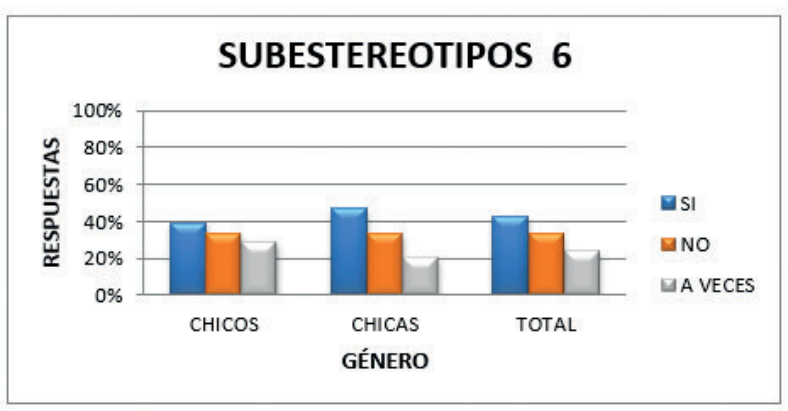

Figura 12. Sub - ítems; diferentes prácticas deportivas en los medios y diferente protagonismo en el deporte de los medios. (6.1, $6.2,6.3,6.4)$. 
En general, la muestra total del alumnado establece que sí existen diferencias con un $57 \%$ frente a los que piensa lo contrario con un $24 \%$.

El análisis de los estereotipos específicos del deporte que aparece en los medios (figura 12) nos muestra los resultados obtenidos por el estereotipo genérico, ya que en ambos casos se deduce la existencia de diferencias en el deporte que aparece en los medios. Por un lado, los chicos opinan que existen estos estereotipos con un 39\% frente a un 33\%. Concretamente, los alumnos apoyan la práctica de ambos géneros según aparezca en los medios, y resaltan el poco protagonismo de las chicas en ellos. Por otro lado, las chicas, también admiten la presencia de estereotipos con $47 \%$ frente a un $33 \%$, y apoyan la opinión de los chicos en la práctica deportiva según los medios e incluso en la falta de protagonismo de ellas mismas. En general, la muestra total nos indica la presencia de este tipo de estereotipos de género con un $43 \%$ frente a un $33 \%$.

\section{Discusión}

En nuestro estudio, al igual que concluyen Fernández-García et al. (2007); Blández et al. (2007) y Gómez-Mármol y De la Cruz Sánchez (2013), las posibles desigualdades de género percibidas en el entorno escolar se asocian con los gustos del profesorado a la hora de planificar y desarrollar sus programaciones. En este sentido, se muestra una tendencia del profesorado a incluir en la programación principalmente actividades consideradas del espacio 'masculino', quedando las actividades del espacio 'femenino' relegadas a un segundo plano. Estos autores también destacan que parece percibirse entre el alumnado de secundaria de que el profesorado "exige" menos a las chicas cuando evalúan cualidades físicas como la fuerza o la resistencia y, por el contrario, es más permisivo con los chicos cuando se evalúan la flexibilidad o actividades como baile o aeróbic. En nuestro estudio parece manifestarse un trato equitativo del profesorado en el trato con el alumnado. El acuerdo mayoritario entre chicos y chicas indica que no se presentan diferencias y que por tanto los docentes son conscientes del papel educador que tienen y de la responsabilidad de formar adecuadamente a sus alumnos y alumnas. Además, el lenguaje sexista en las clases de Educación Física no se confirma puesto que las políticas de igualdad han acercado posturas entre ambos sexos, provocando una exhaustiva vigilancia al maltrato verbal y psicológico que puede acarrear problemas a quienes lo practican. Sin embargo, en la evaluación del alumnado, el docente diferencia el rendimiento de ambos, ya que los chicos sienten que a las chicas se les exige menos para alcanzar la nota mínima para superar los objetivos propuestos en la escuela, provocando que las chicas se acomoden y disminuyan su rendimiento dando lugar a que la sociedad vea que las chicas tienen menor capacidad física creando el estereotipo diferenciado que guarda relación con la evaluación de dicho rendimiento, aunque el alumnado lo niegue de forma general, la conclusión de cada subestereotipo de este ámbito, da lugar a la confirmación de la presencia de dicho estereotipo.

La formación de grupos mixtos tiene sus ventajas y sus inconvenientes, ya que por un lado, viene bien que exista interacción en la actividad física entre ambos géneros para reducir las diferencias entre ambos, pero por otro lado, se crea una comparación que provoca que el alumnado presencie estereotipos en la práctica en grupos mixtos, ya que se visualizan las diferencias en la participación, que provoca un aislamiento del género femenino y el consiguiente comportamiento diferenciable basado en la masculinidad hegemónica, resultados que se asemejan a los presentados por Fernández (2011) y Moreno, Galindo y Villodre (2006). Este tipo de agrupamientos, aunque ofrece diferencias en el rendimiento, favorece la mejora por competir de las alumnas. Por tanto; aunque en estos agrupamientos se produzca una visualización de las posibles diferencias de madurez, rendimiento u otras; es una buena estrategia para motivar y mejorar el rendimiento del alumnado, reduciendo así las distancias entre ambos géneros para lograr alcanzar una igualdad en la actividad física.

Tanto chicas y chicos se manifiestan igual de interesados por la actividad física, aunque se pone de manifiesto como los escolares perciben que los chicos hacen más cantidad de actividad física que las chicas, circunstancia que puede estar condicionada, entre otros factores, por la oferta de actividades en los medios, donde siguen predominando los deportes "masculinos”. Por otra parte, la práctica de actividades físico-deportivas que llevan a cabo las chicas, son fundamentalmente en las actividades individuales, frente a la mayor adscripción de los chicos en las actividades de equipo y de oposición. Por tanto, existe desigualdad en este ámbito, que se confirma con un menor protagonismo en los medios para la práctica de actividad física de las chicas en relación con la estimación de los chicos. En este sentido nuestros resultados se aproximan a lo expuesto por Blández et al. (2007), puesto que, respecto al interés ante la actividad física, parece ser homogéneo en ambos géneros, mostrando interés hacia la práctica. A pesar de esas manifestaciones equitativas, hay algunos estudiantes, tanto chicos como chicas, que piensan que son los varones quienes están más interesados en la práctica de la actividad física. Al igual que concluyen estudios como los de Currie et al. (2004) y Sánchez Chiva-Bartoll y Ruiz-Montero (2016), existe una apreciación general en las chicas y chicos cuando opinan que son los chicos quienes hacen más cantidad de actividad física que las chicas, aunque no ocurre así en otro trabajos como el estudio presentado por Sarkin, McKenzie y Sallis, 1997), que no había diferencias entre el nivel de actividad de actividad física chicos y las chicas de 9 y 10 años.

Los gustos y preferencias de las chicas y de los chicos hacia 
la actividad física son diferentes y están muy vinculados a la percepción que tienen de la misma. En este sentido, todo el alumnado percibe que determinadas actividades físico-deportivas son específicas de género, con lo que, corroborando lo hallado en otros estudios (Meaney y cols., 2002; Moreno et al., 2006), continúan expresando las tipificaciones de género más tradicionales. Así, las actividades físicas que requieren capacidades de: fuerza, y/o resistencia, o son más activas, agresivas o de riesgo (por ejemplo: rugby, fútbol, boxeo, kárate, motociclismo) las vinculan al género masculino. Sin embargo, y en oposición, aquellas actividades más tranquilas o relacionadas con el ritmo, la expresión, la elasticidad, la flexibilidad o la coordinación (por ejemplo: yoga, danza, baile, aeróbic, voleibol, gimnasia artística) las vinculan al género femenino. Lo anterior no hace sino confirmar la persistencia en la asignación de género entre los rasgos instrumentales agentes y expresivos - afectivos tradicionalmente vinculados al espacio de lo "masculino" y lo "femenino" (Crawford y Unger, 2004; Blández et al., 2007). Nosotros hemos podido observar como en los deportes de equipo que suele practicar el alumnado en clase se muestran diferencias entre géneros, aunque los chicos no lo ven así, ya que ellos no lo sienten como las chicas, quienes se sienten discriminadas y aceptan la realidad asumiendo un papel resignado al respecto. La visión del alumno es de indiferencia, que indica inseguridad y miedo en decir la verdad de lo que piensan, pero la sinceridad de las alumnas nos hacer intuir la inferioridad de competitividad y participación que interiorizan en este tipo de deportes el género femenino. Cabe resaltar que el alumnado en general coincide en que cada género no debe ir por separado en la actividad física en general y en la actividad deportiva en particular. Esta coincidencia muestra el camino para conducir y transformar esas desigualdades de manera que exista una equidad entre ambos géneros en el ámbito de la actividad física dentro de la escuela. En nuestro estudio respecto a la expresión corporal se muestra favorable a las chicas, ya que ambos géneros destacan el nivel de ejecución y rendimiento de ellas. Este rendimiento se debe a una mayor práctica y participación del alumnado femenino, provocando una mayor competitividad en este tipo de actividad física, y dando lugar a una discriminación hacia el género opuesto debido a su bajo rendimiento. Por tanto, se deducen diferencias entre chicos y chicas en la expresión corporal, provocando que se considere como actividad física de carácter femenino.

\section{Conclusiones}

En el presente estudio se ha analizado diferentes conductas y percepción ante las posibles desigualdades de género y estereotipos, los cuales, son interiorizados formando conductas diferenciadas. Estas conductas limitan el desarrollo de la per- sonalidad, ya que pretenden guiar el comportamiento de las personas.

Las hipótesis diferenciales entre géneros acertadas, están influidas directamente con un factor común como es el rendimiento. Dicho factor nos muestra patrones diferenciales entre géneros, ya que las chicas muestran un mayor rendimiento en actividades expresivas y de menor competitividad, y que por el contrario los chicos destacan en su rendimiento en actividades competitivas y de mayor esfuerzo físico. En la práctica de todos estos tipos de actividades se presenta un mejor autoconcepto de los chicos sobre su propio rendimiento, competitividad y participación con respecto a las chicas. Esta diferencia tan evidente nos parece indicar que no se han puesto en práctica muchas iniciativas para solventar estas desigualdades, todo ello debido a la complejidad de las relaciones causa - efecto de la gran diversidad de factores que influyen en dicho rendimiento. Un ejemplo de estos factores es la experiencia en la actividad física, la cual, si fuese equitativa en ambos géneros se reducirían las diferencias. Otros de los factores que establecen las diferencias entre ambos géneros dentro de los ítems analizados son; la participación desigual, debido a que las expectativas que mantienen las chicas sobre su participación e implicación en la actividad física y el deporte, difieren de las de los chicos, siendo mayor la de estos; el diferente interés hacia la Educación Física, donde las chicas conceden el suficiente el valor y la utilidad a la asignatura, aunque existen diferencias de género, y son los chicos quienes puntúan más alto en estas variables; la comparativa desigual, donde las chicas se diferencian levemente de sus compañeros, pero los chicos muestran su ego manteniendo el rechazo de las chicas hacia la competición y los resultados; y la competitividad diversa en Educación Física que tiene cada género, debido a que existen diferencias significativas con la percepción que mantienen los chicos, que se sienten bastante más capaces y hábiles que las chicas, aunque la percepción que mantienen las chicas no es negativa.

Se puede afirmar que persisten creencias relacionadas con la actividad física y el deporte. Se sigue vinculando a los chicos con actividades que requieran fuerza, resistencia, que son más activas, agresivas o de riesgo, como los deportes de equipo convencionales (fútbol, baloncesto, balonmano, etc.) mientras que a las chicas se las sigue vinculando con actividades de ritmo, expresión, elasticidad, flexibilidad y coordinación. Estas creencias confirman la formación de un modelo, del que hablan muchos autores, sobre diferencias biológicas, donde se destaca "la fuerza masculina" y "la debilidad femenina" con la que se formó el área de educación física en la escuela. Dicho modelo ha sido una de las fuentes en la educación que ha mantenido los estereotipos de género, y por tanto, dicho agente debe ser quien corrija o transforme estas malas actitudes de género, a través de prácticas equitativas. Además, dicho modelo condiciona que ciertas actividades físicas sean 
relacionadas con un género exclusivamente, y por tanto, limitando la participación del género opuesto en este tipo de actividades.

Podemos concluir de manera general cómo queda de ma- nifiesto la existencia de muchos estereotipos entre hombres y mujeres en la muestra que participa en dicho estudio, que dan a entender diferencias, conduciendo que se cumplan la mayoría de las hipótesis planteadas en la investigación.

\section{Referencias}

1. ALADINO (2016). Estudio de Vigilancia del Crecimiento, Alimentación, Actividad Física, Desarrollo Infantily Obesidad en España 2015. Agencia Española de Consumo, Seguridad Alimentaria y Nutrición. Ministerio de Sanidad, Servicios Sociales e Igualdad. Madrid. Disponible en: http://www.aecosan.msssi.gob.es/AECOSAN/docs/documentos/nutricion/observatorio/Estudio_ALADINO_2015.pdf

2. Alvariñas-Villaverde, M. y Novoa-Pérez, A. (2015). Pensamientos relacionados con la actividad física y el género en adolescentes de Galicia. Sportis. Scientific Journal of School Sport, Physical Education and Psychomotricity, 2(1), 23-35. https://doi.org/10.17979/sportis.2016.2.1.1439

3. Alvariñas, M., Fernández-Villarino, M. A. y López-Villar, C. (2009). Actividad física y percepciones sobre deporte y género. Revista de investigación en educación, 6, 113-122.

4. Blández, J., Fernández, E., y Sierra, M.A. (2007). Estereotipos de género, actividad física y escuela: La perspectiva del alumnado. Revista de curriculum y formación del profesorado, 11(2), 1-21.

5. Castillo, O., Romero, S., González, T., \& Campos, M. (2012). Gender equity in physical education: The use of information. Sex Roles, 67,108121. doi: 10.1007/11199-011-0103-5

6. Cervelló, E., Jiménez, R., Del Villar, F., Ramos, L., \& Santos-Rosa (2004). Goal orientations, motivational climate, equality, and discipline of Spanish physical education students. Perceptual and Motor Skills, 99, 271-283.

7. Currie, C., Roberts, C., Morgan, A., Smith, R., Settertobulte, W., Samdal, O., \& Rasmussen, V. (Eds.). (2004). Young people's health in context. Health Behaviour in School-aged Children (HBSC) Study: International Report from the 2001/2002 Survey. Copenhagen: Health Policy for Children and Adolescents (HEPCA) (4). World Health Organization Regional Office for Europe. Disponible en: http://www.euro.who.int/__data/ assets/pdf_file/0008/110231/e82923.pdf?ua=1

8. Fernández, I., Canet O., \& Giné-Garriga M. (2017). Assessment of physical activity levels, fitness and perceived barriers to physical activity practice in adolescents: cross-sectional study. European Journal of Pediatrics. 176(1), 57-65. doi: 10.1007/00431-016-2809-4.

9. Fernández, E., Blández, J., Camacho, MJ., Sierra, MA., Vázquez, B., Rodriguez, I., Mendizabal, S., Sánchez, F., \& Sánchez, M. (2007): Estudio de los estereotipos de género vinculados con la actividad física y el deporte en los centros docentes de Educación Primaria y Secundaria: Evolución y vigencia. Diseño de un programa integral de acción educativa
(2004-2007). Instituto de la mujer. Ministerio de Igualdad. Disponible en: http://www.inmujer.gob.es/centroDocMujer/abnetcl.exe/O7007/ ID $415 \mathrm{~d} 5 \mathrm{~d} 08 / \mathrm{NT} 1$

10. Frömel, K; Formánková, S., \& Sallis, J. F. (2002). Physical Activity and Sport Preferences of 10 to 14 Year-Old Children: A 5-Year Prospective Study. Acta Univ. Palaki Olomuc. Gymn, 32(1), 11-16.

11. Gómez-Mármol, A. y De la Cruz Sánchez, E. (2013). Diferencias de género y de nivel académico en la utilidad percibida de la educación física escolar. Journal of Sport and Health Research, 5(2), 193-202.

12. Lamas, M. (2000). Diferencias de sexo, género y diferencia sexual. Cuicuilco, $7(18), 1-24$

13. López, F., Lara, A., Espejo, N., \& Cachón, J. (2016). Influencia del género, la edad y el nivel de actividad física en la condición física de alumnos de educación primaria. Revisión Bibliográfica. Retos: nuevas tendencias en educación física, deporte y recreación, 29, 129-133.

14. Moreno, J.A., Galindo, C., \& Villodre, N. (2006). Actitudes hacia la práctica físico-deportiva según el sexo del practicante. Revista internacional de ciencias del deporte, 2(3), 20-43.

15. Piedra, J., García-Pérez, R., Rebollo, M.A., \& Ries, FJ. (2011). Actitudes hacia la coeducación en Andalucía ¿Es el profesorado de Educación Física diferente? Revista de Investigación en Educación, 9(2), 200-208

16. Secchi, J., García, G., España-Romero, V., \& Castro-Piñero, J. (2014). Physical fitness and future cardiovascular risk in argentine children and adolescents: an introduction to the ALPHA test battery. $A r$ chivos argentinos de pediatría, 112(2), 132-140. doi: 10.1590/032500752014000200005

17. Soler, S. (2006). Actitudes y relaciones de niñas y niños ante contenidos de la educación física en primaria estereotipados por el género: el caso del fútbol. En: Vázquez, B. (Coord.), Las mujeres jóvenes y las actividades físico deportivas. Madrid: Consejo Superior de Deportes, 119-150.

18. Valdivia, P.A., López, M., Lara, A., \& Zagalaz, M.L. (2012). Concepto de coeducación en el profesorado de Educación Física y metodología utilizada para su trabajo. Movimento, 18(4), 197-217.

19. Vázquez, B., Fernández, E., \& Ferro, S. (2000): Educación Física y género: Modelo para la observación y el análisis del comportamiento del alumnado y del profesorado. Madrid: Gymnos.

20. Williams, J. E., \& Best, D. L. (1990). Measuring Sex Stereotypes: A multination study, Newbury Park, Calif.: Sage. 